Revue internationale P.M.E.

Économie et gestion de la petite et moyenne entreprise

\title{
Allocation de ressources, avantage concurrentiel et performance des petites et moyennes entreprises de l'industrie manufacturière française
}

\section{Jacques-Bernard Sauner-Leroy}

Volume 15, numéro 1, 2002

URI : https://id.erudit.org/iderudit/1008801ar

DOI : https://doi.org/10.7202/1008801ar

Aller au sommaire du numéro

Éditeur(s)

Presses de l’Université du Québec

ISSN

0776-5436 (imprimé)

1918-9699 (numérique)

Découvrir la revue

Citer cet article

Sauner-Leroy, J.-B. (2002). Allocation de ressources, avantage concurrentiel et performance des petites et moyennes entreprises de l'industrie manufacturière française. Revue internationale P.M.E., 15(1), 65-85.

https://doi.org/10.7202/1008801ar
Résumé de l'article

L'analyse des liens entre la possession de un ou plusieurs avantages concurrentiels et la performance des firmes est un domaine qui a déjà été assez largement abordé dans la littérature de management stratégique. Certains de ces travaux ont cherché plus précisément à mettre en évidence des modes de combinaisons de ressources conduisant à la réalisation de niveaux de performance élevés. Toutefois, leurs champs d'investigation sont demeurés le plus souvent restreints à des domaines d'activité spécifiques, de sorte que les résultats auxquels ils aboutissent ne peuvent être généralisés. Partant de ce constat et s'appuyant sur le modèle de " ressources de base " qui apparaît comme le corpus théorique le plus complet sur cette question, l'objet de cette étude est de mettre en évidence, à partir de l'exploitation de 3049 enquêtes issues de la base de données de la Banque de France sur les comportements économiques et financiers des PME de l'industrie manufacturière française, des profils types de combinaison de ressources et de création d'avantages concurrentiels et de les comparer sur plusieurs critères de performance. Pour ce faire, une analyse typologique est menée. Elle conduit à la mise en évidence de six stratégies types de combinaisons de ressources associées à des avantages concurrentiels différents. Les résultats des tests effectués sur les comparaisons de performance entre ces six groupes indiquent que les entreprises qui obtiennent les meilleurs résultats sont celles qui adoptent un positionnement concurrentiel différencié fondé sur une approche qui situe le client au cœur de leur stratégie et qui utilisent principalement la fonction marketing comme support de création de leurs avantages compétitifs. 


\title{
Allocation de ressources, avantage concurrentiel et performance des petites et moyennes entreprises de l'industrie manufacturière française
}

\author{
Jacques-Bernard SAUNER-LEROY
}

Banque de France

MOTS CLÉS

\section{Combinaison de ressources - Avantages concurrentiels \\ Performances - Modèle « de ressources de base » \\ PME - Industrie manufacturière \\ Typologie de stratégies d'allocation de ressources}

\begin{abstract}
RÉSUMÉ
L'analyse des liens entre la possession de un ou plusieurs avantages concurrentiels et la performance des firmes est un domaine qui a déjà été assez largement abordé dans la littérature de management stratégique. Certains de ces travaux ont cherché plus précisément à mettre en évidence des modes de combinaisons de ressources conduisant à la réalisation de niveaux de performance élevés. Toutefois, leurs champs d'investigation sont demeurés le plus souvent restreints à des domaines d'activité spécifiques, de sorte que les résultats auxquels ils aboutissent ne peuvent
\end{abstract}

\section{L'AUTEUR}

JaCQUES-BERnARD SAUNER-Leror est adjoint de direction à la Banque de France, a été responsable au sein de la Direction des entreprises de la gestion et de l'exploitation de la base de données sur les comportements économiques et financiers des PME de l'industrie manufacturière. II est actuellement détaché à la Commission européenne en tant qu'expert national auprès de la Direction générale économique et financière où il est en charge de l'exploitation de la base $\mathrm{BACH}$ et des questions touchant aux PME et à l'entrepreneuriat. Adresse : Banque de France, 49-1477 GME, 75049 Paris cedex 01, France. Courriel : <jacques-bernard.saunerleroy@cec.eu.int>. 
être généralisés. Partant de ce constat et s'appuyant sur le modèle de « ressources de base " qui apparaît comme le corpus théorique le plus complet sur cette question, l'objet de cette étude est de mettre en évidence, à partir de l'exploitation de 3049 enquêtes issues de la base de données de la Banque de France sur les comportements économiques et financiers des PME de l'industrie manufacturière française, des profils types de combinaison de ressources et de création d'avantages concurrentiels et de les comparer sur plusieurs critères de performance. Pour ce faire, une analyse typologique est menée. Elle conduit à la mise en évidence de six stratégies types de combinaisons de ressources associées à des avantages concurrentiels différents. Les résultats des tests effectués sur les comparaisons de performance entre ces six groupes indiquent que les entreprises qui obtiennent les meilleurs résultats sont celles qui adoptent un positionnement concurrentiel différencié fondé sur une approche qui situe le client au cœur de leur stratégie et qui utilisent principalement la fonction marketing comme support de création de leurs avantages compétitifs.

\begin{abstract}
The analysis of the links between the possession of one or more competitive advantages and the performance of the firms is a topic which has been already largely broached in strategic management literature. Some of these studies have focused more precisely on the resource combinations leading to high performance levels. Generally speaking, however, their fields of investigation have remained restricted to specific industries, with the result that the findings cannot be applied broadly. Taking this observation as a starting point, and using the "ResourceBased View » model, which represents the most complete theoretical corpus on this question, this study aims to identify standard profiles of resource combinations and of competitive advantage creation and to compare their respective performances. The analysis makes use of 3,049 surveys taken from the Banque de France database on the economic and financial behaviour of SME's in the French manufacturing sector. For that purpose, a typological analysis is led. Six standard strategies of resource combinations associated with different competitive advantages are thus brought to the fore. The six groups are then compared on three performance criteria. It is shown in particular that firms that obtain the best performances are those that adopt a differentiated competitive positioning using the marketing function as a support of creation of their competitive advantages.
\end{abstract}

\title{
RESUMEN
}

Numerosos estudios sobre estrategia empresarial se han interesado en el vínculo entre la existencia de una o varias ventajas competitivas y el buen desempeño de las empresas. Algunos de esos estudios se han concentrado más precisamente en las combinaciones de recursos que conducen a niveles de resultados superiores en un sector dado. Ahora bien, en la mayoría de los casos, los campos de investigación de esos estudios se han limitado a sectores de actividad específicos, por lo cual no es posible generalizar sus conclusiones. Partiendo de este hecho, y utilizando 3.049 encuestas provenientes de la base de datos del Banco de Francia, relativas a los comportamientos económicos y financieros de las PyMEs de la industria manufacturera francesa, el presente estudio tiene por objeto evidenciar

Revue internationale P.M.E., vol. 15, $\mathrm{n}^{\circ}$ 1, 2002 
perfiles tipo, con diferentes combinaciones de recursos y su influencia en la creación de ventajas competitivas, para posteriormente compararlos en función de sus efectos en el rendimiento de las empresas. Se observa, en particular, que las empresas que ostentan los mejores resultados son aquéllas que adoptan una postura competitiva claramente diferenciada, basada en un enfoque en donde el cliente se sitúa en el eje mismo de la estrategia y que utilizan principalmente la función marketing como elemento de apoyo para la lograr ventajas competitivas.

\section{ZUSAMMENFASSUNG}

Die Analyse der Zusammenhänge zwischen dem Besitz von mehreren Konkurrenzvorteilen und der Unternehmensleistung ist ein Bereich, der schon genügend behandelt wurde in der Literatur des strategischen Managements. Einige von diesen Arbeiten haben versucht, die Kombinationsformen von Ressourcen, die zur Realisierung von höheren Leistungen führen, genauer hervorzuheben. Ihr Forschungsbereich ist jedoch meistens steckengeblieben in spezifischen Aktivitätsbereichen, so dass die Resultate nicht verallgemeinert werden können. Ausgehend von dieser Feststellung und aufbauend auf dem Modell 'ResourceBased View' als geeignetes Theoriegerüst, wurde eine Studie durchgeführt. Ziel dieser Studie war es auf der Basis von 3049 Untersuchungen von Daten der Banque de France, das ökonomische und finanzielle Verhalten der französischen KMU in der Industrie zu erfassen. Dabei sollten Profiltypen von Ressourcenkombinationen herausgefunden werden, welche Konkurrenzvorteile begründen. Diese Kombinationen wurden dann in der Folge mit verschiedenen Leistungskriterien verglichen. Zu diesem Zweck wurde eine analytische Typologie durchgeführt. Sie führte auf die Hervorhebung von sechs strategischen Kombinationstypen von Ressourcen, verbunden mit verschiedenen Konkurrenzvorteilen. Die Resultate der durchgeführten Tests wurden auf einem Leistungsvergleich zwischen den sechs Gruppen aufgebaut. Die Unternehmen mit den besten Ergebnissen sind jene, welche ihre differenzierte Konkurrenzposition auf einen Ansatz aufbauen, der die Kunden ins Zentrum ihrer Strategie stellt. Dabei wurde in erster Linie die Funktion des Marketings als Unterstützung für die Begründung ihrer Wettbewerbsvorteile eingesetzt.

\section{Introduction}

Depuis les travaux de Porter (1980, 1985), il est communément admis que les différentes stratégies que les firmes, notamment industrielles, poursuivent peuvent se décliner en trois catégories types que sont les stratégies de coûts - ou de volume -, les stratégies de différenciation et les stratégies de niche. À ces stratégies types sont fréquemment associés, à travers l'analyse de la chaîne de valeur, de multiples modes d'allocation ou de combinaison de ressources adaptées à la poursuite de l'avantage concurrentiel recherché par la firme. Ainsi, par exemple, pour une stratégie de volume, la minimisation des coûts de production pourra être obtenue, entre autres, par une automatisation accrue de la chaîne de fabrication, par une meilleure organisation logistique ou encore par le recours à la sous-traitance ou à la délocalisation de tout ou partie de la production.

Revue internationale P.M.E., vol. 15, nº 1, 2002

(C) 2002 - Presses de l'Université du Québec

Édifice Le Delta I, 2875, boul. Laurier, bureau 450, Sainte-Foy, Québec G1V 2M2 • Tél. : (418) 657-4399 - www.puq.uquebec.ca

Tiré de : Revue internationale P.M.E., vol. 15, $\mathrm{n}^{\circ} 1$, sous la direction de Pierre-André Julien. 
Pour autant, alors que l'ensemble de ces concepts et préconisations sont extrêmement bien balisés dans la littérature d'analyse stratégique, notamment celle dite de vulgarisation, il est frappant de constater que les travaux empiriques portant sur la question de l'efficacité relative des stratégies types et, au sein de celles-ci, de l'efficacité relative des diverses combinaisons de ressources possibles, sont demeurés rares, surtout en ce qui concerne le comportement des petites et moyennes entreprises.

En outre, les études qui ont été menées sur ce sujet sont restées confinées soit à l'analyse de domaines d'activité particuliers - avec des auteurs comme Hitt et Ireland (1985), Hall (1994) ou Walsh et al. (1996) -, soit à l'analyse d'avantages spécifiques ou d'une compétence donnée (Arregle, 1995) de sorte qu'il n'existe pas de «modèle » suffisamment général permettant de déceler les modes d'allocation de ressources les plus efficaces pour les firmes industrielles. Cet état de fait peut s'expliquer par la conjugaison de deux éléments. D'une part, dans la mesure où les ressources et aptitudes sont souvent diffuses au sein de l'organisation (Dierickx et Cool, 1989; Barney, 1991), leur appréhension ne peut s'effectuer autrement que par une analyse détaillée de leurs modes d'organisation et de coordination au sein de l'entreprise (Baden-Fuller et Stopford, 1992 ; Stalk, Evans et Schulman, 1992). D'autre part, l'accès à ce type d'informations sur un panel élargi d'entreprises est particulièrement difficile à obtenir.

La base de données de la Banque de France sur les comportements stratégiques des petites et moyennes entreprises de l'industrie manufacturière française permet toutefois de lever partiellement ce double obstacle en ce qu'elle contient des indications, à un niveau de détail relativement fin, sur les modes d'allocation de ressources de plus de 4000 entreprises d'effectifs compris entre 40 et 2000 salariés.

L'objectif de cette étude est donc, à partir de l'exploitation d'un échantillon de 3206 PME issu de cette base de données, de mieux cerner les différents critères qui peuvent être à l'origine de la formation de la rentabilité des firmes industrielles et de mettre en exergue les stratégies d'allocation d'actifs les plus efficientes ou, à l'inverse, les moins performantes.

Cet article s'organise en trois parties. La première présente une synthèse de la littérature consacrée aux liens entre les allocations de ressources, la création d'avantages concurrentiels et la performance des firmes. La deuxième partie aborde la méthodologie qui a été retenue et la troisième partie analyse les principaux résultats. Les conclusions tendent à indiquer que les combinaisons de ressources les plus efficaces sont celles qui s'inscrivent dans le cadre de stratégies de différenciation fondées sur une approche qui situe le client au cœur de leurs préoccupations et qui utilisent principalement la fonction marketing comme support de création de leurs avantages compétitifs.

Revue internationale P.M.E., vol. 15, nº 1, 2002 


\section{Allocation de ressources, avantages concurrentiels et performance: un lien bien établi dans la littérature mais encore peu testé empiriquement ou de façon partielle}

Depuis la fin des années 1980, avec la remise en cause du paradigme stratégique jusqu'alors dominant qui relie la performance d'une entreprise à sa capacité d'adaptation à son environnement et le développement d'un nouveau courant insistant sur l'importance des ressources comme support du positionnement concurrentiel des firmes, un certain nombre d'études ont mis en évidence le fait que l'explication des différences de profitabilité entre les entreprises doit davantage être recherchée au niveau de la mise en œuvre des moyens et compétences disponibles dans chaque firme plutôt qu'au niveau des caractéristiques des secteurs d'activité (Buzzel et Gale, 1987; Rumelt, 1991; Rocquebert, Phillips et Westfall, 1996; MacGahan et Porter, 1997). Plusieurs auteurs ont ainsi pu démontrer que la détention de un ou plusieurs avantages concurrentiels constituait la principale source de réalisation de niveaux de profitabilité élevés au sein d'une industrie donnée (Hambrick, 1983; White, 1986; Calori et Ardison, 1988; Baden-Fuller et Stopford, 1992).

Dès lors, il est désormais généralement admis que pour expliquer les causes des performances hétérogènes constatées entre les firmes, il est nécessaire de s'interroger sur la façon dont celles-ci parviennent à créer un avantage concurrentiel et à le maintenir durablement.

Selon Porter (1980, 1985), la nature et l'ampleur de l'avantage compétitif créé résultent de la position relative de la firme en termes de coûts ou de différenciation par rapport à ses concurrents. Dans ce schéma, la performance est la traduction des actions engagées par les dirigeants par le choix des meilleurs « pilotes » de la chaîne de valeur dans leur activité. Ainsi, dans le cadre d'une stratégie de maitrise des coûts, il convient d'identifier pour chaque fonction les facteurs qui permettent de réduire les coûts : économies d'échelle, synergies entre plusieurs activités, localisation géographique... C'est en maîtrisant mieux que les concurrents tous les facteurs de la chaîne qui déterminent la valeur de l'offre aux yeux des clients que l'entreprise se crée son avantage.

Remettant en cause ce schéma «porterien », sans pour autant s'y opposer fondamentalement (Arregle, 1996), les tenants de la théorie des ressources - ou du «management par les ressources»-postulent, quant à eux, que l'avantage concurrentiel provient essentiellement de l'utilisation par l'entreprise d'actifs, de compétences ou de procédés qui lui sont propres, car souvent diffus et fortement imprégnés de la culture de l'entreprise et, par conséquent, difficilement transférables, imitables ou substituables (Dierickx et Cool, 1989). Plus précisément, c'est la combinaison efficace de ces ressources qui est à l'origine de la construction de l'avantage concurrentiel (Grant, 1991), cette faculté à coordonner diverses 
compétences constituant ce que Prahalad et Hamel (1990) ont dénommé les «compétences centrales» de l'entreprise. Le modèle «de ressources de base » (RDB) issu de ces travaux mais offrant une synthèse des approches environnementale et interne des déterminants de la performance des firmes, considère ainsi chaque entreprise comme une collection originale d'actifs et de compétences physiques ou intangibles, de sorte qu'aucune firme n'est semblable à une autre. Les compétences propres à chaque firme proviennent, selon ce modèle, de l'expérience et des savoir-faire acquis, de la mise en place de processus ou routines spécifiques ou encore de l'existence d'une culture organisationnelle particulière. Associées aux actifs tangibles ou intangibles accumulés au cours du temps, ces compétences sont directement reliées par le modèle RDB à la performance de la firme, pour autant qu'elles contribuent à la création d'un avantage concurrentiel, c'est-à-dire le fait de posséder des ressources permettant d'accroître la qualité ou la compétitivité de son offre. Le modèle RDB, de la sorte, relie les compétences internes de la firme aux conditions de son environnement externe et stipule que les entreprises les plus performantes sont celles qui possèdent un stock de ressources (compétences et actifs tangibles ou intangibles) le plus approprié aux caractéristiques du marché et qui procèdent à l'allocation optimale ou efficiente de ces ressources (Collis et Montgomery, 1995).

Plusieurs recherches ont ainsi visé à tester empiriquement la nature des liens entre la possession de ressources distinctives, la création d'avantages concurrentiels et la performance. Hitt et Ireland (1985) ont notamment mis en évidence le fait que, dans l'industrie des biens d'équipement, les firmes les plus profitables étaient celles qui avaient accru l'automatisation de leur production, obtenu de leurs fournisseurs des composants plus fiables et moins chers ou assuré un meilleur contrôle des coûts, de la qualité et des délais de production. Hall (1994) met en avant, à partir d'études de cas, le rôle clé de certaines variables sur la performance, telles que la réputation, le savoir-faire ou la culture d'entreprise. Walsh et al. (1996) montrent l'importance de la maîtrise de l'accès aux matières premières dans le secteur des semi-conducteurs. Powell et Dent-Micallef (1997) insistent, quant à eux, sur les compétences humaines et la relation avec les fournisseurs pour expliquer de meilleurs niveaux de performance liés à l'introduction des technologies de l'information dans le secteur du commerce de détail. D'autres études se sont concentrées sur les facteurs à l'origine de la création d'avantages concurrentiels particuliers comme, par exemple, l'innovation technologique (Gaffard, 1990a et 1990b) ou les services autour des produits (Mendel, 1991). Certains auteurs se sont également préoccupés de repérer les fonctions de l'entreprise se situant à la source de leur compétitivité. Chrisman et Boulton (1987) identifient ainsi les fonctions R-D, production, marketing et distribution. L'ensemble de ces travaux, toutefois, se sont focalisés sur l'étude soit de secteurs d'activité très ciblés, soit d'avantages ou de compétences spécifiques, de sorte qu'ils ne peuvent prétendre à une validité d'ordre général (Arregle, 1995). Aucune recherche portant sur les domaines de compétence 
à l'origine de la création des avantages concurrentiels n'a encore été réalisée sur l'industrie dans son ensemble ou alors que de façon partielle. Very et Arregle (1995) ou Arregle (1995) se sont efforcés d'adopter une approche plus générale, mais leur analyse ne concerne qu'une partie seulement des secteurs de l'industrie manufacturière française ou ne tient compte que d'une source potentielle de création d'avantages concurrentiels (en l'occurrence la fonction production pour Arregle, 1995), sans pour autant établir de conclusions en termes d'efficacité des combinaisons de ressources mises en évidence.

\section{Méthodologie}

Pour mener à bien une telle recherche, il est nécessaire d'utiliser des informations sur une population d'entreprises représentatives de l'industrie. La base de données de la Banque de France sur les comportements stratégiques des entreprises de l'industrie manufacturière française, alimentée par voie d'enquête auprès de plus de 4000 PME sur deux années, répond à cette exigence, le taux de représentativité, mesuré en termes d'effectifs employés, atteignant en moyenne plus de $20 \%$.

La population étudiée comprend à l'origine 3738 PME de l'industrie manufacturière française dont les effectifs sont compris entre 35 et 499 salariés ${ }^{1}$ et qui ont été enquêtées en 1996 et 1997 par des agents de la Banque de France spécialement formés à cet effet. Ces derniers ont utilisé un questionnaire assisté par ordinateur pour recueillir les réponses des chefs d'entreprise à environ 150 questions posées au cours d'un entretien en face à face et portant à la fois sur les caractéristiques de la firme (mode de production, organisation interne), de son management (objectifs, formalisation de la prise de décision, horizon temporel de la stratégie), de son environnement (turbulence sectorielle, forces concurrentielles) ainsi que sur son positionnement concurrentiel (parts de marché, avantages concurrentiels, fonctions support des avantages concurrentiels) et son mode de croissance (croissance externe et alliances). Ce faisant, les traitements statistiques opérés ne font que refléter les réponses, qui sont le plus souvent des réponses de perception, apportées par le chef d'entreprise à des questions relatives à sa stratégie passée ou présente. En ce sens, les résultats présentés doivent s'interpréter comme s'insérant dans le cadre d'une analyse «statique », c'est-à-dire arrêtée à un moment donnée, des stratégies mises en œuvre par les entreprises interrogées.

S'inspirant assez largement du modèle « RDB », l'analyse a été conduite dans le but de mettre en évidence les principales combinaisons de ressources types mises en œuvre par les PME de l'industrie manufacturière française et de les comparer

1. Cette étude porte donc uniquement sur les entreprises enquêtées qui répondent à la définition de la PME retenue par l'INSEE, à l'exclusion des firmes enquêtées de plus grande taille.

Revue internationale P.M.E., vol. 15, $\mathrm{n}^{\circ}$ 1, 2002 
sur un certain nombre de critères de performance. De fait, les variables retenues se rapportent aux avantages concurrentiels développés par l'entreprise (prix, qualité, performance technique et innovation, image de marque, services associés, délais ou souplesse, proximité géographique) ${ }^{2}$ ainsi qu'aux fonctions mises en œuvre citées par les chefs d'entreprise comme étant à l'origine de la création de ces avantages. Seules huit fonctions ont été retenues parce qu'elles correspondent à celles mentionnées dans la littérature comme sources principales de la compétitivité des firmes industrielles : production, R-D, marketing, commercialisation (Chrisman et Boulton, 1987; Very et Arregle, 1995; Arregle, 1995), gestion des ressources humaines (Blais, 1996; Castro, Guerin et Lauriol, 1998), services (Caves et Porter, 1977; Mendel, 1991), approvisionnement et, enfin, logistique (Very et Arregle, 1995 et Arregle, 1995). Cette méthode de sélection des variables est ainsi conforme à ce que Ketchen et Shook (1996) considèrent être la plus pertinente lorsqu'on a recours à une analyse typologique.

Concernant les avantages concurrentiels, les chefs d'entreprise étaient amenés à se positionner, pour chacun d'entre eux, sur une échelle de valeur variant de 1 («position très défavorable par rapport aux principaux concurrents ») à 5 («position très favorable par rapport aux principaux concurrents »). Quant aux fonctions mises en œuvre, il leur était demandé de préciser celles qui leur permettaient principalement de soutenir leur position concurrentielle sur les marchés en les classant de 1 (la principale) à 5 (la cinquième par ordre d'importance). Les fonctions ainsi sélectionnées par le chef d'entreprise étaient par la suite détaillées de façon à ce qu'il puisse indiquer plus précisément les aspects de chaque fonction à l'origine de la création des avantages concurrentiels de sa firme. La liste de ces compétences « de base » (63 au total) a été établie à partir des travaux de Porter (1985), Stevenson (1976) et Hitt et Ireland (1985).

La méthodologie retenue s'est attachée, dans un premier temps, à faire émerger des typologies de combinaison de fonctions mises en œuvre et d'avantages concurrentiels poursuivis puis, à travers une analyse descriptive de chaque groupe, de caractériser plus précisément les compétences distinctives détenues. Une comparaison des groupes sur différents critères de performance permet, dans un second temps, d'isoler les profils de combinaison de ressources les plus efficaces. À l'issue de la phase de traitement statistique des données, en raison de l'existence de données manquantes, la population finale comprend 3049 entités.

Une analyse factorielle (analyse en composantes principales avec rotation varimax) permet de dégager les facteurs sous-jacents aux variables d'origine et d'éliminer celles qui apportent peu d'informations ou qui se révèlent peu robustes.

2. Cette liste des avantages concurrentiels a été élaborée à partir des travaux de Porter par des professeurs universitaires de premier plan en management stratégique.

Revue internationale P.M.E., vol. 15, nº 1, 2002 
Le recours à cette technique présente l'avantage de permettre de s'affranchir d'éventuels problèmes de multicolinéarité dans les variables retenues pour l'analyse typologique (Punj et Stewart, 1983). À la fin de cette procédure, six facteurs issus du regroupement de douze variables émergent. Plus de 66,5\% de l'information initiale est ainsi restituée, témoignant d'un bon niveau de consistance du modèle (annexe I). Une analyse typologique, confirmée par une analyse discriminante, conduite sur ces six facteurs (nuées dynamiques, puis classification ascendante hiérarchique sur les groupes stables avec utilisation de la méthode de Ward ${ }^{3}$ ), permet ensuite de faire apparaître des groupes d'entreprises présentant des comportements proches en termes d'allocation de ressources et d'avantage concurrentiel ${ }^{4}$. Une analyse descriptive complète enfin la description de chacun de ces groupes, notamment au niveau du détail des fonctions à l'origine de la création des avantages concurrentiels. Cette analyse descriptive, réalisée par une procédure d'analyse de variance (test de Ducan), permet, en outre, d'asseoir la validité des clusters, dans la mesure où ils servent à confirmer les caractéristiques propres à chacun d'entre eux (Aldenderfer et Blashfield, 1984 ; Anderberg, 1973 ; Ketchen et Shook, 1996).

\section{Résultats et discussion}

\subsection{Analyses typologique et descriptive}

Le tableau 1 présente la synthèse des résultats obtenus à l'issue des analyses typologique et descriptive.

Six groupes de PME manufacturières ayant des caractéristiques homogènes en termes de compétences centrales et d'avantage concurrentiel sont ainsi mis en évidence. Le tableau intègre également les traits distinctifs propres à chaque groupe qui sont issus de l'analyse descriptive. Le détail des résultats statistiques ayant servi de base à la constitution de ce tableau est présenté en annexes I et II.

Le premier groupe (562 cas) comprend des entreprises qui développent leur position concurrentielle principalement autour de la fonction «gestion des ressources humaines » et secondairement autour de la fonction «production». Aucun avantage concurrentiel n'est véritablement identifié comme tel. Cela étant, la «qualité » est le critère le plus souvent recherché. Les éléments descriptifs des

3. Le critère de Ward est utilisé, car il n'y a pas d'a priori sur le nombre d'observations attendues dans chaque cluster d'une part, et parce que la nature des variables retenues dans l'analyse (échelles de réponse variant de 1 à 5) rend improbable l'existence de valeurs extrêmes pouvant perturber la qualité du modèle (Ketchen et Shook, 1996).

4. La population est suffisamment large pour que l'on puisse considérer que la critique formulée par Jardine et Sibson (1971) sur l'instabilité de la solution en cas d'étroitesse de l'échantillon ne s'applique pas ici.

Revue internationale P.M.E., vol. 15, n ${ }^{\circ} 1,2002$

(C) 2002 - Presses de l'Université du Québec

Édifice Le Delta I, 2875, boul. Laurier, bureau 450, Sainte-Foy, Québec G1V 2M2 • Tél. : (418) 657-4399 - www.puq.uquebec.ca

Tiré de : Revue internationale P.M.E., vol. 15, n 1, sous la direction de Pierre-André Julien. 


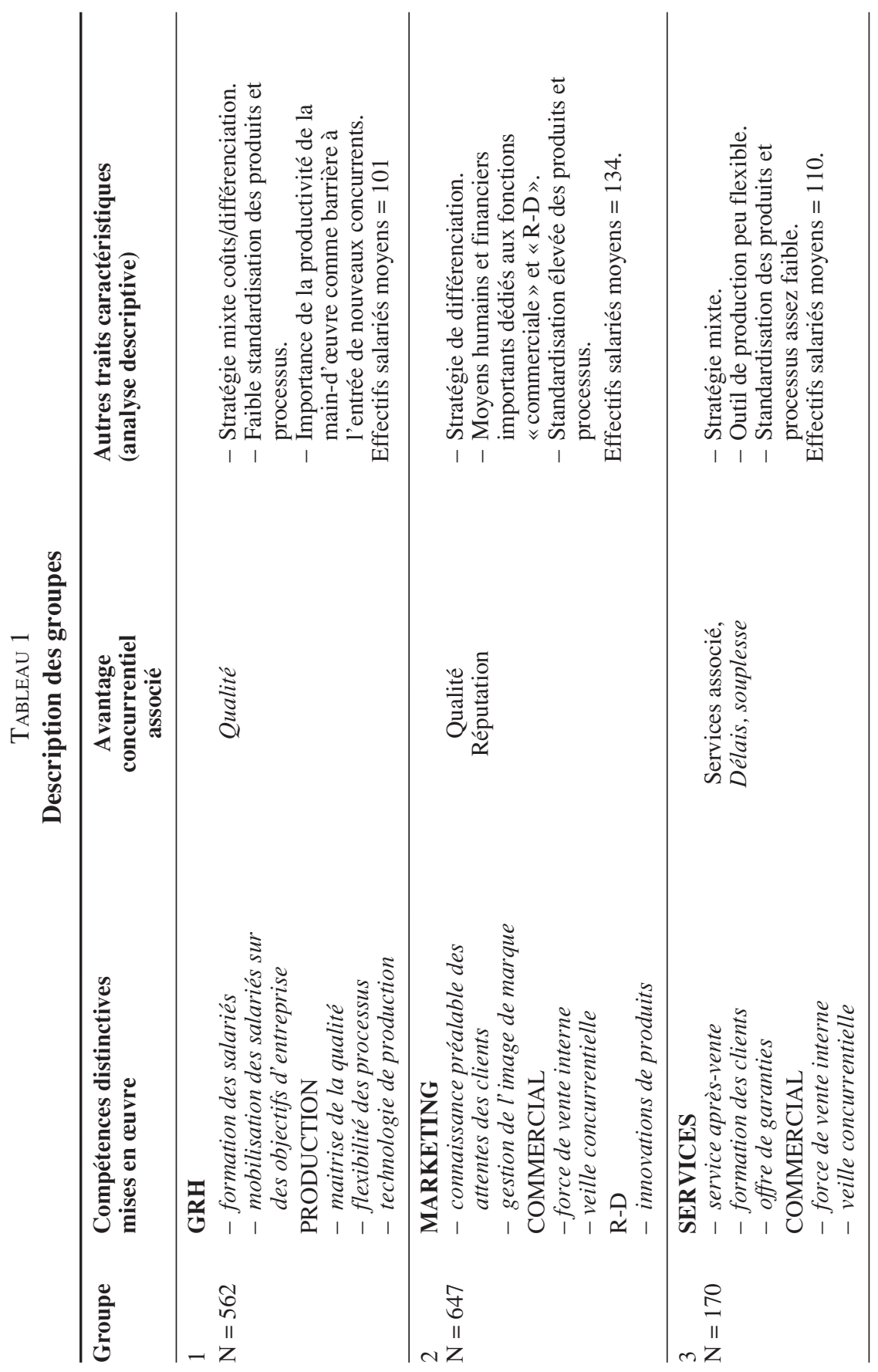

Revue internationale P.M.E., vol. 15, nº 1, 2002 


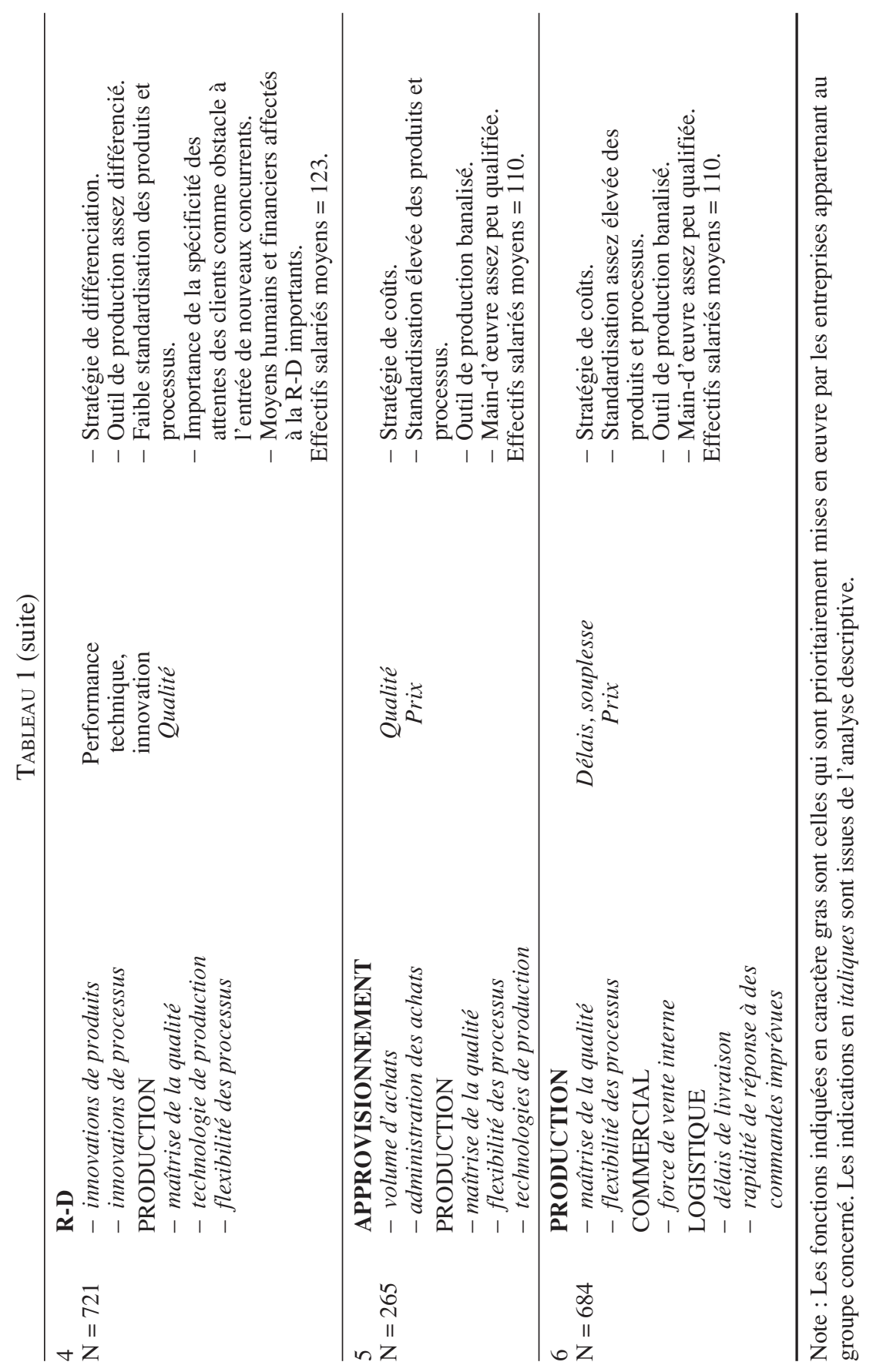

Revue internationale P.M.E., vol. 15, nº 1, 2002 
ressources mises en œuvre semblent indiquer que ces firmes poursuivent une stratégie d'efficience (compétivité-prix reposant sur des facteurs autres que les économies d'échelle ou les effets d'expérience) fondée sur la productivité de la main-d'œuvre. Ainsi, la fonction GRH dans ces entreprises semble répondre à la description faite par Coriat (1995) du «modèle de la compétence » en ce qu'elle met l'accent sur la recherche de l'implication des salariés dans leurs activités de travail. L'idée que seul un fort engagement de ces derniers puisse conduire à la qualité des produits, à la flexibilité de l'organisation et à la maîtrise des coûts constitue le fondement d'une telle démarche (Castro et al., 1998). Ce groupe de firmes est surreprésenté dans l'industrie du papier-carton, de l'industrie métallurgique et de l'industrie de fabrication de matériels de transport terrestre. Par contre, il est sousreprésenté dans l'industrie chimique ainsi que dans les industries de fabrication des machines et équipements, du caoutchouc et des matières plastiques et des équipements électriques et électroniques.

Le deuxième groupe (647 cas) comprend des entreprises pour lesquelles la réputation et la qualité de l'offre constituent des avantages concurrentiels forts. Compte tenu de l'importance de la fonction marketing dans la définition des avantages concurrentiels ainsi que de la gestion de l'image de marque et / ou de la réputation ou encore des efforts déployés en matière commerciale à travers l'existence de forces de ventes internes, ce groupe peut être caractérisé comme fortement « orienté clients ». L'importance également attachée à l'aspect « veille concurrentielle » semble indiquer que ces firmes cherchent à positionner leur offre par rapport à celle de la concurrence. À bien des égards, ces firmes s'apparentent aux «bâtisseurs rentables » décrits par Gertz et Baptista (1996) en ce qu'elles mettent l'accent, dans leur stratégie, sur la gestion de l'actif clients, sur leur capacité à développer de nouveaux produits, sur la valorisation de leur «capital marque » ou encore sur la comparaison de la valeur de l'offre par rapport à celle des concurrents. Au niveau sectoriel, ce groupe est surreprésenté dans l'industrie chimique. Par contre, il est sous-représenté dans l'industrie métallurgique et dans la fabrication de matériels de transport terrestre.

Le troisième groupe (170 cas) rassemble des entreprises qui développent très nettement leurs avantages concurrentiels sur la prestation de services associés à leur offre. Ce groupe de firmes se caractérise par la définition d'une offre globale, de style «package », dans laquelle, en reprenant la typologie proposée par Furrer (1997), le service associé aux produits vendus constitue soit une source de différenciation (Dornier, 1990), soit un élément de l'offre attendu par les clients et fonctionnant dès lors comme une barrière à l'entrée (Bain, 1965 ; Caves et Porter, 1977). L'analyse descriptive tend à démontrer que, des trois types de services identifiés par Lele (1986), les firmes de ce groupe en offrent essentiellement deux : rapidité de réponse aux besoins des clients (avantage concurrentiel délais, souplesse)

Revue internationale P.M.E., vol. 15, nº 1, 2002 
et réduction des risques des clients (service après-vente, offre de garanties). Les entreprises du groupe 3 peuvent ainsi être caractérisées comme des «orientées services ». Elles sont surreprésentées parmi les industries de fabrication de machines et équipements, de fabrication d'équipements électriques et électroniques et de matériel de transport. Par contre, elles sont sous-représentées dans les secteurs du papier-carton et du caoutchouc et des matières plastiques.

Le quatrième groupe (721 cas) comprend des entreprises qui s'appuient sur leur fonction R-D ainsi que, de façon plus secondaire, sur leur fonction «production » pour développer des avantages concurrentiels en termes de performance technique et d'innovation. En s'appuyant notamment sur les travaux de Gaffard (1990a et 1990b), on peut considérer que ces entreprises deviennent innovatrices grâce à l'incorporation des unités de R-D dans le processus de production. Elles sont ainsi susceptibles, en acquérant de nouvelles compétences pour développer de nouveaux marchés, d'obtenir des avantages compétitifs sur ces marchés (Boucher, 1995). Ce groupe, qualifié «d'innovateur», est surreprésenté dans les industries de fabrication de machines et équipements et de matériels de transport terrestre. Il est sous-représenté dans les industries agroalimentaires.

Le cinquième groupe (265 cas) est composé de firmes pour lesquelles les fonctions «production» et surtout « approvisionnement» se situent au cœur de la création de leurs avantages concurrentiels, lesquels sont plutôt orientés vers la qualité, sans qu'il s'agisse pour elles d'un véritable facteur de compétitivité par rapport à leurs principaux concurrents. De même que pour le prix, il s'agit plus d'un avantage recherché que d'un avantage effectif. Les caractéristiques de ces entreprises indiquent que celles-ci s'inscrivent dans le cadre d'une stratégie de type «orientation produit» (Arregle, 1995). Ce groupe est surreprésenté parmi les industries agroalimentaires, du cuir et de la chaussure et de la transformation du bois. Il est par contre sous-représenté dans l'industrie des équipements électriques et électroniques et dans l'industrie métallurgique.

Le sixième groupe (684 cas) rassemble des entreprises qui développent des avantages concurrentiels axés sur les délais et la souplesse à partir des fonctions «logistique» et «production». Au sein de celles-ci, les aspects essentiels mis en œuvre indiquent clairement que la compétitivité repose sur une flexibilité des processus de production décrits par Gerwin et Tarondeau (1984) et Tarondeau (1999) comme celle permettant, notamment, « de s'adapter aux variations de la demande sans créer de stocks ou de retards et de s'adapter aux variations non anticipées dans les inputs provenant de l'extérieur ». Ce groupe est surreprésenté parmi les industries du caoutchouc et des matières plastiques, des produits minéraux et de la métallurgie. Il est sous-représenté parmi les industries de fabrication de machines et équipements, d'équipements électriques et électroniques et de matériels de transport terrestre.

Revue internationale P.M.E., vol. 15, n 1,2002 


\subsection{Comparaison des performances des groupes}

La performance permettant d'apprécier la pertinence des modes d'allocation de ressources et de la solidité des avantages concurrentiels créés peut s'analyser selon trois critères principaux : des critères d'ordre financier, des critères d'ordre opérationnel et des critères mesurant l'efficacité globale (Venkatraman et Ramanujam, 1986).

Deux indicateurs financiers et un indicateur d'efficacité globale permettent de reprendre cette distinction en trois types de mesure de la performance :

- d'une part, un premier indicateur financier, le taux de marge brute d'exploitation a été retenu, car il fournit une estimation du niveau de profitabilité de la firme et, donc, une estimation de son pouvoir de marché. En effet, plus ce dernier est élevé, plus la marge prise par la firme peut être importante et plus sa profitabilité est forte. On retrouve ici la notion de mark-up, définie comme la capacité pour une firme de fixer ses prix au-dessus de ses coûts marginaux du fait de l'exercice d'un pouvoir de marché (Martins, Scarpetta et Pilat, 1996);

- d'autre part, un deuxième indicateur financier, la rentabilité brute du capital d'exploitation, rapport entre la marge brute d'exploitation et le capital d'exploitation, permet d'exprimer une efficacité de type opérationnel dans la mesure où ce ratio fournit une estimation de l'efficience dans la mise en œuvre du capital employé dans le cycle productif, donc des ressources propres à la firme.

Ces deux premiers indicateurs présentent en outre l'avantage de bien correspondre à la logique du modèle «RDB » puisqu'ils synthétisent l'un, une performance reliée aux conditions externes de la firme, c'est-à-dire son environnement concurrentiel et l'autre, une performance reliée aux facteurs internes à la firme, c'est-à-dire ses ressources et compétences.

Toutefois, afin de lisser les effets conjoncturels, la comparaison des groupes a été effectuée sur la moyenne sur quatre ans de ces deux $\operatorname{ratios}^{5}$ (soit les années 1994, 1995, 1996 et 1997). Il paraît important de procéder à un tel lissage pour au moins deux raisons. En premier lieu, parce que la marge brute d'exploitation, dénominateur commun à ces deux ratios, est assez largement influencée par les évolutions du cycle conjoncturel ; le recours à une moyenne de ratios permet de réduire ce biais. En second lieu, certaines firmes exercent leurs activités dans des secteurs dans lesquels la variabilité des résultats peut être importante (secteurs «cycliques»). Ici encore, le recours à une moyenne de ratios permet de réduire l'influence de ces aspects.

5. Ces ratios ont également été bornés afin de limiter l'influence des valeurs extrêmes.

Revue internationale P.M.E., vol. 15, no 1, 2002 
Enfin, troisième critère de performance retenu, un indicateur non financier qualitatif permet d'apprécier l'efficacité globale de la firme dans la poursuite de sa stratégie. Cet indicateur permet de juger du degré d'adéquation entre les compétences développées en interne par la firme et les attentes du marché. Il s'agit donc d'un indicateur orienté vers une performance « de marché », c'est-à-dire mesurant la capacité de la firme à répondre aux exigences du marché quant aux caractéristiques fondamentales de l'offre. Cela peut être directement relié à la notion de valeur attachée à une ressource : une ressource n'a de valeur stratégique que si elle contribue à la production d'une offre à un prix que les clients sont prêts à payer (Collis et Montgomery, 1995). Pour construire un tel indicateur, les réponses aux questions relatives aux facteurs clés de succès dans l'activité et aux avantages concurrentiels développés par l'entreprise ont été utilisées de façon à calculer une «position concurrentielle » définie comme une note comprise entre 0 (non-adéquation entre les facteurs clés de succès et les avantages concurrentiels) et 100 (parfaite adéquation entre les facteurs clés de succès et les avantages concurrentiels).

La comparaison sur ces trois types d'indicateurs (performance financière, performance de marché) a été effectuée par analyse de variance (test de Duncan) ou par tests non paramétriques (test U de Mann-Whitney). Le tableau 2 présente les résultats obtenus.

TABLEAU 2

Comparaison des groupes sur les indicateurs de performance

\begin{tabular}{lcccccc}
\hline Stratégie & $\begin{array}{c}\text { Orientation } \\
\text { d'efficience } \\
(\mathbf{1})\end{array}$ & $\begin{array}{c}\text { Orientation } \\
\text { clients } \\
(\mathbf{2})\end{array}$ & $\begin{array}{c}\text { Orientation } \\
\text { services } \\
(\mathbf{3})\end{array}$ & $\begin{array}{c}\text { Innovateurs } \\
\text { (4) }\end{array}$ & $\begin{array}{c}\text { Orientation } \\
\text { produit } \\
(\mathbf{5})\end{array}$ & $\begin{array}{c}\text { Flexibilité } \\
\text { productive } \\
(\mathbf{6})\end{array}$ \\
\hline $\mathrm{RBCE}$ & $14,05 \%(=)$ & $15,98 \%(+)$ & $14,70 \%(=)$ & $14,74(=)$ & $13,66(-)$ & $12,84(-)$ \\
\hline $\mathrm{TMBE}$ & $6,99 \%(=)$ & $8,25 \%(+)$ & $6,98 \%(=)$ & $7,54 \%(+)$ & $6,17(-)$ & $6,82 \%(=)$ \\
\hline $\begin{array}{l}\text { Performance } \\
\text { de marché* }\end{array}$ & $75,52(=)$ & $76,63(=)$ & $77,67(+)$ & $76,42(=)$ & $71,4(-)$ & $73,4(-)$ \\
\hline
\end{tabular}

Notes : Le signe (+) indique que la moyenne est significativement supérieure, au seuil de $5 \%$, à celle des autres groupes.

Le signe (-) indique que la moyenne est significativement inférieure, au seuil de $5 \%$, à celle des autres groupes.

Le signe (=) indique que la moyenne n'est pas significativement différente de celle des autres groupes de même signe $(=)$.

* Les moyennes de cet indicateur varient selon un intervalle assez étroit. Cela tient au fait que les chefs d'entreprise étaient amenés à se positionner sur une échelle qualitative présentant elle-même des intervalles de réponse étroits (minimum : 1 ; maximum : 5). En outre, la distribution des réponses aux questions utilisées dans cet indicateur est marquée par une certaine concentration sur les valeurs les plus élevées $(3,4$ ou 5$)$, d'où la tendance au resserrement des valeurs du score calculé. 
La lecture des résultats inscrits dans le tableau 2 fait nettement ressortir la stratégie «d'orientation clients » comme la plus performante, notamment en termes de profitabilité et de rentabilité. Ce constat apparaît d'ailleurs cohérent avec des résultats issus d'autres recherches (notamment Kostecki, 1994) ou avec des conclusions effectuées par des consultants en stratégie d'entreprise (Gertz et Baptista, 1996). Ces firmes sont en moyenne de plus grande taille que les autres. On constate toutefois que cette différence de taille tient, au moins en partie, à la mise en œuvre de moyens plus importants affectés aux fonctions «commercial» et «R-D », en cohérence avec l'importance attachée aux compétences distinctives développées.

Parmi les autres constats que l'on peut établir à la lecture du tableau 2, il y a le fait que la stratégie de coûts est généralement ${ }^{6}$ la moins performante des stratégies menées dans l'industrie manufacturière française, à l'inverse des stratégies de différenciation. Cela avait d'ailleurs déjà pu être mis en évidence dans des études précédentes utilisant la même base de données, mais sur une période d'observation différente (Durand, 1997; Sauner-Leroy, 1997).

Les «innovateurs » présentent des niveaux de performance financière très honorables, confirmant ainsi les résultats de travaux portant sur l'impact de l'innovation sur la profitabilité (Geroski, Machin et Van Reenen, 1993; Montgomery, 1995). Leur performance demeure cependant en retrait par rapport à celle des « orientés clients ». Les caractéristiques comparées des outils de production de ces deux groupes laissent supposer qu'une explication à cet état de fait pourrait résider dans le degré de standardisation des produits et procédés, lequel est plus élevé chez les « orientés clients », autorisant ces derniers à produire à coût unitaire plus faible, donc de manière plus profitable. Ce constat est par ailleurs cohérent avec celui établi par Hitt et Ireland (1985) dont l'une des conclusions était que les firmes ayant la meilleure profitabilité contrôlaient mieux leurs coûts de production grâce, notamment, à une automatisation accrue de leurs procédés.

Par ailleurs, le groupe des « orientés services » se distingue par la conjugaison d'une position concurrentielle (ou performance de marché) particulièrement élevée et d'une performance financière moyenne. Il est ainsi probable que les firmes de ce groupe aient tendance à «surinvestir» sur les caractéristiques de leur offre répondant aux critères privilégiés par le marché, ce qui pèserait sur leur profitabilité. Cette analyse s'inscrit dans la lignée de résultats d'autres travaux soulignant que les effets de la différenciation induite par le service tendraient à s'estomper avec le temps, alors que le client percevrait toujours le service comme un élément incontournable de l'offre, obligeant par là même chaque concurrent à investir dans

6. Le terme « généralement » est employé, car les résultats portent sur une moyenne d'un groupe de firmes. Ils ne sont donc pas nécessairement pertinents pour une entreprise du groupe prise isolément, qui peut dégager des niveaux élevés de profitabilité.

Revue internationale P.M.E., vol. 15, nº 1, 2002 
ce type de prestation pour rester dans le marché. Le service évoluerait ainsi du statut d'avantage concurrentiel à celui de barrière à l'entrée, et sa rentabilité diminuerait (Lele, 1986; Bowen, Siehl et Schneider, 1989).

\section{Conclusion}

Cette étude, qui s'appuie sur un échantillon large de PME, a permis de mettre en évidence six comportements types de management stratégique dans l'industrie manufacturière française auxquels peuvent être associés des niveaux de performances financières et de marché différents. Les conclusions auxquelles elle aboutit mériteraient d'être testées à nouveau sur un échantillon aussi étendu mais sur une périodicité différente afin de les valider entièrement. Il n'en demeure pas moins que, du fait même de la profondeur des données, les résultats de cette étude permettent d'apporter un éclairage instructif sur les stratégies d'allocation de ressources des PME industrielles françaises. En particulier, il ressort très nettement que les entreprises qui obtiennent les meilleures performances sont celles qui adoptent un positionnement concurrentiel différencié fondé sur une approche qui situe le client au cœur de leur stratégie et qui utilisent principalement la fonction marketing comme support de création de leurs avantages compétitifs. Ce constat rejoint celui qui a pu être établi par ailleurs par des praticiens du conseil en management à partir d'études de cas de grandes sociétés américaines, ce qui tendrait à indiquer que ce mode de développement constitue une voie privilégiée de la performance. Il ne s'agit pas pour autant de l'ériger en un modèle type à destination des managers ou des cabinets d'audit, d'autres stratégies pouvant se révéler mieux adaptées aux spécificités d'une entreprise ou d'un secteur d'activité donnés. À cet égard, un prolongement de cette étude consisterait à rechercher, au sein de chacun des six groupes identifiés, les critères organisationnels ou stratégiques distinctifs des entreprises les plus performantes.

Revue internationale P.M.E., vol. 15, nº 1, 2002 


\section{AnNeXe I \\ Analyse factorielle Description des facteurs}

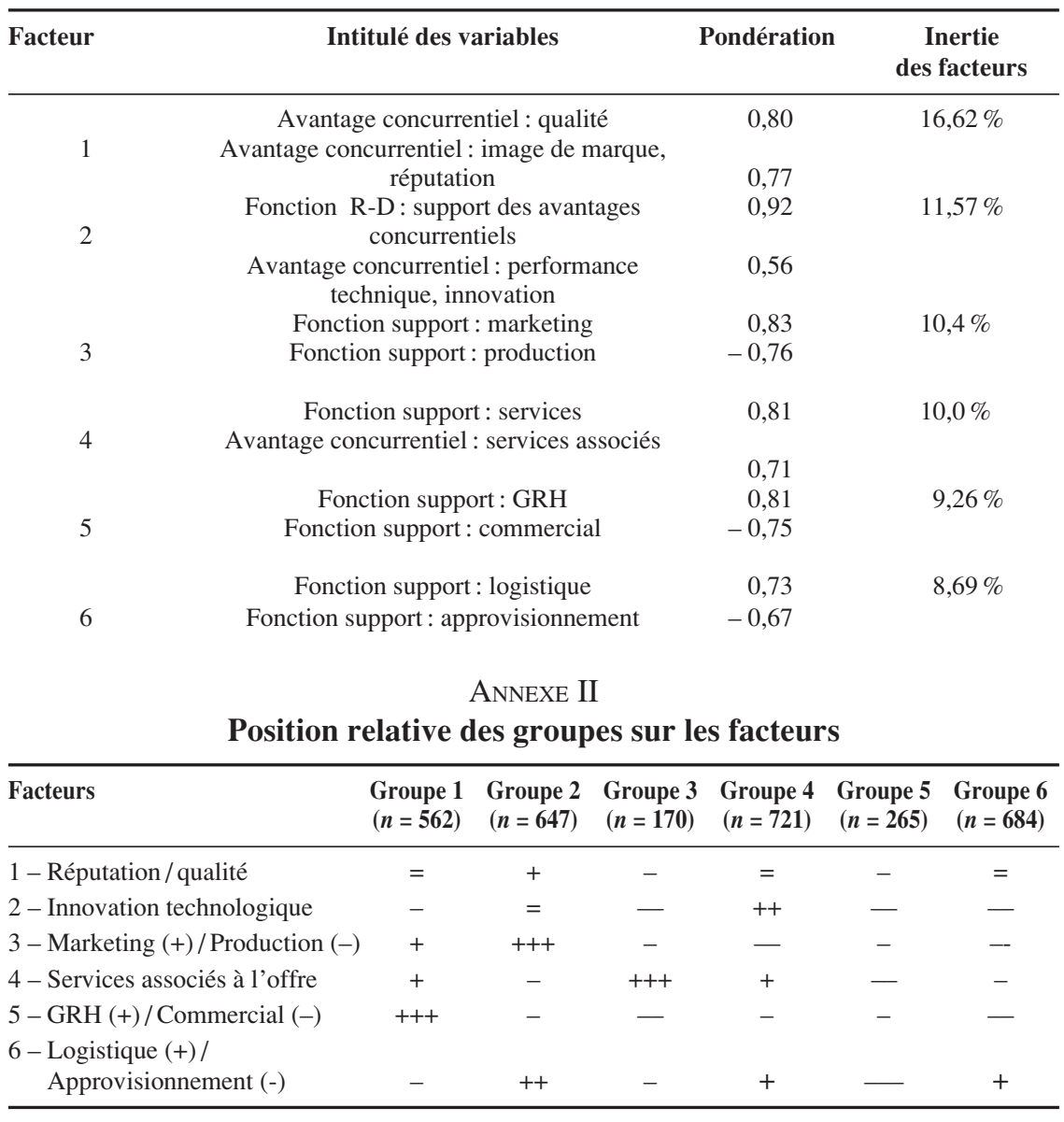

Notes : Les signes $(+++)(++)$ et $(+)$ indiquent respectivement que la moyenne est très significativement, significativement et assez significativement supérieure, au seuil de $5 \%$, à celle des autres groupes. Les signes $(-)(-)$ et $(-)$ indiquent que la moyenne est très significativement, significativement et assez significativement inférieure, au seuil de $5 \%$, à celle des autres groupes.

Le signe (=) indique que la moyenne est significativement supérieure à celles mentionnées (-), mais également significativement inférieure à celles mentionnées (+).

\section{Bibliographie}

Aldenderfer, M. et R. Blashfield (1984), Cluster Analysis, Newbury Park, CA, Sage. Anderberg, M. (1973), Cluster Analysis for Applications, New York, Academic Press. 
ARREGLE, J.L. (1995), «Le savoir et l'approche Resource Based: une ressource et une compétence », Revue française de gestion, septembre-octobre.

ARREGLE, J.L. (1996) « Analyse Resource Based et identification des actifs stratégiques », Revue française de gestion, mars-avril.

BADEN-Fuller, C.W et J.M STOPFORD (1992), Rejuvenating the Mature Business, Londres, Routledge.

BAIN, J.S. (1965), Barriers to New Competition: Their Character and Consequences in Manufacturing Industries, Cambridge, Harvard University Press.

BLAIS, R. (1996), Les ressources humaines : l'atout concurrentiel, Québec, Presses Inter Universitaires.

BARNEY, J. (1991), «Firm resources and sustained competitive advantage », Journal of Management, vol. 17, p. 99-120

BOUCHER, L. (1995) «La diffusion de l'innovation technique au sein de l'entreprise : le rôle des ressources humaines », Revue française de gestion, mars-avril-mai.

BOwEN, D.E., C. SIEHL et B. SCHNEIDER (1989), «A framework for analysing customer service orientations in manufacturing », Academy of Management Review, vol. 14, $\mathrm{n}^{\mathrm{o}} 1$.

Buzzel, R.D et B.T Gale (1987), The PIMS Principles, New York, Free Press.

CALORI, R. et J.M. ARDISON (1988), « Les stratégies de différenciations dans les impasses concurrentielles », Revue française de gestion, $\mathrm{n}^{\circ} 68$.

CAStro, J.L., F. Guerin et J. LAURIOL (1998), «Le modèle des “3 C” en question », Revue française de gestion, mars-avril-mai.

CAVES, R.E. et M.E. PORTER (1977), « From entry barriers to mobility barriers : conjectural decisions and contrived deterrence to new competition », Quarterly Journal of Economics, mai.

Chrisman, J.J. et W.R. Boulton (1987), «An exploratory study of business-level skills and how they influence strategic performance », Academy of Management Proceedings.

Collis, D. et C. Montgomery (1995), «Competing on resources : strategy in the 1990's », Harvard Business Review, juillet-août, p. 118-128.

CORIAT, B. (1995), «Les méthamorphoses de la division du travail dans la crise contemporaine : l'implication et ses modèles », dans J. Bidet et J. Texier, La crise du travail, Paris, Presses universitaires de France.

DIERICKX, I. et K. COOL (1989), «Asset stock accumulation and the sustainability of competitive advantage », Management Science, vol. 35, n 12.

DORNIER, P.P. (1990), «Émergence d'un management de l'après-vente », Revue française de gestion, juin-juillet-août.

DURAND, R. (1997), Management stratégique des ressources et performance des firmes, Thèse, H.E.C., Jouy-en-Jossas.

FURRER, O. (1997), «Le rôle stratégique des services autour des produits », Revue française de gestion, mars-avril-mai.

GAFFARD, J.L. (1990a), Économie industrielle et de l'innovation, Paris, Dalloz.

Revue internationale P.M.E., vol. 15, $\mathrm{n}^{\circ}$ 1, 2002 
GAFFARD, J.L. (1990b), «Innovations et changements structurels : revue critique de l'analyse moderne de l'innovation et des changements structurels », Revue d'économie politique, $\mathrm{n}^{\mathrm{0}} 3$.

GEROSKI, P., S. MACHIN et J. VAN REENEN (1993), « The profitability of innovating firms », Rand Journal of Economics, vol. 24.

GeRTZ, D. et J. BAPTISTA (1996), Croître, un impératif pour l'entreprise, Paris, Village mondial.

GERWIn, D. et J.C. TARONDEAU (1984), «La flexibilité dans les processus de production: le cas de l'automobile », Revue française de gestion, $\mathrm{n}^{\circ} 46$.

GRANT, R.M. (1991), «The resource-based theory of competitive advantage : implications for strategy formulation », California Management Review, vol. 33.

HALL, R. (1994), « A framework for identifying the intangible sources of sustainable competitive advantage », dans G. Hamel et A. Heene, Competence-based Competition, New York, John Wiley and Sons.

HAMBRICK, D.C. (1983), «High profit strategies in mature capital goods industries : a contingency approach », Academy of Management Review, vol. 26.

HITT, M.A. et R.D. IRELAND (1985), « Corporate distinctive competences, strategy, industry and performance », Strategic Management Journal, vol. 6, nº 3, p. 273-293.

JARDINE, N. et R. SIBSON (1971), Mathematical Taxonomy, New York, Wiley.

KetChen, D. et C. SHooK (1996), « The application of cluster analysis in strategic management research : an analysis and critique », Strategic Management Journal, vol. 17, p. 441-458.

KosteCKI, M. (1994), « Nouvelles tendances du marketing des services », Revue française du marketing, vol. 149, $\mathrm{n}^{\circ} 4$.

LELE, M.M. (1986), « How service needs influence product strategy », Sloan Management Review, automne.

MAcGahan, A. et M.E. PorTer (1997), « How much does industry matter, really?», Strategic Management Journal, vol. 18.

MARTINS, J.O., S. SCARPETTA et D. PILAT (1996), « Mark-up pricing, market structure and the business cycle», OECD Economic Studies, n 27.

MENDEL, D. (1991), «Marketing international et management des services : cas des entreprises industrielles », dans H. Mathe, Managing Services Across Border, CergyPontoise, Eurolog Press.

MONTGOMERY, C. (1995), «Of diamonds and rust», dans C. Montgomery, Resource-Based and Evolutionary Theories of the Firm, Boston, MA, Kluwer.

PORTER, M.E. (1980), Competitive Strategy: Techniques of Analyzing Industries and Competitors, New York, Free Press.

PORTER, M.E. (1985), Competitive Advantage : Creating and Sustaining Superior Performance, New York, Free Press.

Revue internationale P.M.E., vol. 15, $\mathrm{n}^{\circ}$ 1, 2002 
Powell, T.C. et A. DenT-Micallef (1997), «Information technology as competitive advantage : the role of human, business and technology resources », Strategic Management Journal, vol. 18.

PRAHALAD, C.K. et G. HAMEL (1990), « The core competence of the corporation », Havard Business Review, mai-juin.

PunJ, G. et W. STEWART (1983), «Cluster analysis in marketing research : Review and suggestion for application », Journal of Marketing Research, vol. 20, p. 134-148.

Rocquebert, J.A., R.L. PhilliPS et P. WestFall (1996), « Market versus management : what drives profitability? », Strategic Management Journal, vol. 17.

RuMELT, R. (1991), « How much does industry matter? », Strategic Management Journal, vol. 12.

SAUNER-Leroy, J.B. (1997), «Stratégie et formation de la rentabilité des PMI : une approche empirique », Bulletin de la Banque de France, n 39, mars.

StalK, G., P. Evans et L.E. Schulman (1992), «Competing on capabilities : the new rules of corporate strategy », Harvard Business Review, mars-avril.

Stevenson, H.H. (1976), « Defining corporate strengths and weaknesses », Sloan Management Review, vol. 17.

TAROndeAu, J.C. (1999), «Approches et formes de la flexibilité », Revue française de gestion, mars-avril-mai.

VenKatraman, N. et V. RAmAnujam (1986), « Measurement of business performance in strategic research », Academy of Management Review, vol. 11, p. 801-814.

Very, P. et J.L. ARREgLe (1995), «Combinaisons de ressources et avantage concurrentiel », Actes de la IVe Conférence internationale de management stratégique, Paris.

Walsh, S., R. Boylan, J. Morone et A. PAulson (1996), « Core capabilities and strategy, empirical evidence for the semiconductor silicon industry », dans H. Thomas et D. O’Neil, Strategic Integration, Chichester, John Wiley and Sons.

WHITE, R.E. (1986), « Generic business strategies, organisational context and performance : an empirical investigation », Strategic Management Journal, vol. 7.

Revue internationale P.M.E., vol. 15, n $\mathrm{n}^{\circ} 1,2002$ 